\section{Fixed drug combinations}

\author{
Per Hartvig Honoré
}

The interest in drug combinations has varied over time. In earlier times, pharmacists would possibly put a range of active ingredients in the same preparation which may have contained active remedies to treat the heart, the lungs and muscle pain. Multi-ingredient pill preparations were presented to the patient in a nice box and taken once or twice daily. Dosages were adjusted to patient needs and compliance was supported by easy administration. Over time, such preparations were regarded as non-hygienic, time consuming to prepare and costly.

Pharmaceutical industry developments such as 'one dose to all' meant that fixed combinations became less usable. However, many pharmaceutical preparations, particularly for pain, contained analgesic substances and other ingredients such as spasmolytics, sedatives and caffeine. In the late 1970s, the authorities tidied up the flora of fixed drug combinations by claiming that ingredients acting by different mechanisms for the indication should be given in sufficient and effective doses and have a similar duration of action. It was considered important that there should be no doubt about when a new dose should be taken and not just when one of the substances no longer had any effect. Adverse effects could appear if a new dose was taken at this early time point. However, a breakthrough of

Correspondence to Professor Per Hartvig Honoré, Department of Drug Design and Pharmacology, University of Copenhagen, Universitetsparken 2, Copenhagen 2100, Denmark; peh@sund.ku.dk symptoms might occur when the new dose was taken if some components were largely eliminated and therefore ineffective. As a result, the number of analgesics fell drastically and there became a general reluctance from the drug industry to market fixed drug combinations. The result of this was a large increase in the number of drugs prescribed for an individual.

In the early 1980s older patients took three to four drugs; today they may be taking 10 when self-medication preparations are included. In turn, this increases the demand for compliance support, more individual drug information on each preparation, interaction problems and the growth of clinical pharmacy to cope with the new challenges.

Therefore, what is the issue? No single pharmaceutical substance has only one target. Generally, there are two, three, or more often, several acting directly or indirectly. For example, phenothiazine antipsychotic drugs have an action on dopaminergic, cholinergic, serotonergic, noradrenergic/adrenergic, histamine and sodium channel receptors. The antipsychotic effect is weak but the side effects are distressing for the patient. Remarkably, the drug with the most interactions on different receptors, clozapine, is regarded as the most clinically effective of the class. The reason for this is not understood but a certain delicate balance of all receptor interactions may furnish the best cocktail for clinical efficacy. Other drugs with a multitude of receptor interactions are antidepressants, opioids, benzodiazepines, digitalis, among many others. Still it seems that a broad receptor interaction as in the older antidepressant seems more favourable for clinical success and the anxiolytic effect of morphine is still unbeaten. In conclusion, a single drug substance may provide a fixed drug combination with many pharmacological effects.

Have we overlooked the importance of a balanced treatment in an unbalanced disease state? Disease can be described as an imbalance in a normal system that should be corrected. The body does not often have the instructions or ability to repair an imbalance. A single drug may not have the ability to counterbalance the event, perhaps even less so if there is a single target of action. A combination of actions as in many drug substances and preparations might be more successful to reach the balance. However, an individualised dosing regimen for a patient will be lost with a fixed dose combination, irrespective of how it is provided.

In the present issue of European Journal of Hospital Pharmacy there is an account of the advantages and drawbacks of fixed drug combinations. We are interested in your opinions and comments in the journal.

\section{Competing interests None.}

Provenance and peer review Commissioned; internally peer reviewed.

To cite Hartvig Honoré P. Eur J Hosp Pharm 2014;21:1

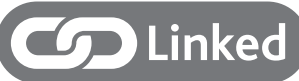

http://dx.doi.org/10.1136/ejhpharm-2013-000390

Eur J Hosp Pharm 2014;21:1.

doi:10.1136/ejhpharm-2013-000389 